\title{
Robotic Incisional Hernia Repair After Robotic-assisted Radical Prostatectomy (RARP): A 3-port Approach
}

\author{
HSIEN-CHE OU ${ }^{1,2}$, LI-HUA HUANG ${ }^{1}$, KUANG-HSI CHANG ${ }^{3,4,5}$, YEN-CHUAN OU ${ }^{1,2,3}$, MIN-CHE TUNG ${ }^{1}$,

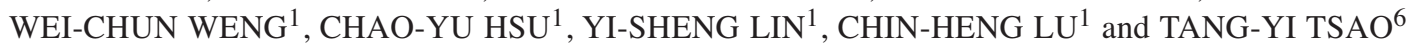 \\ ${ }^{1}$ Division of Urology, Department of Surgery, Tungs' Taichung Metro Harbor Hospital, Taichung, Taiwan, R.O.C.; \\ ${ }^{2}$ Post-graduate Year Training (PGY), Department of Medical Education, Taipei Veterans General Hospital, \\ School of Medicine, National Yang-Ming University, Taipei, Taiwan, R.O.C.; \\ ${ }^{3}$ Department of Research, Tungs' Taichung Metro Harbor Hospital, Taichung, Taiwan, R.O.C.; \\ ${ }^{4}$ Graduate Institute of Biomedical Sciences, China Medical University, Taichung, Taiwan, R.O.C.; \\ ${ }^{5}$ General Education Center, Jen-Teh Junior College of Medicine, Nursing and Management, Miaoli, Taiwan, R.O.C.; \\ ${ }^{6}$ Department of Anatomical Pathology, Tungs' Taichung Metro Harbor Hospital, Taichung, Taiwan, R.O.C.
}

\begin{abstract}
Background/Aim: Incisional hernia is a complication that occurs occasionally, and surgical intervention is required to prevent more severe sequela. While there are several options for management, robotic-assisted incisional repair has not been well discussed yet. We herein report a case series of 10 patients who underwent roboticassisted incisional hernia repair (RIHR) after robotic-assisted radical prostatectomy (RARP). The aim of the study was to examine the feasibility of incisional hernia repair with da Vinci ${ }^{\circledR}$ robotics. Patients and Methods: We recruited patients from a group of 2,000 consecutive patients who underwent RARP from December, 2005 to June, 2020 by a single surgeon. Patient characteristics included age, body mass index (BMI), PSA level, pathology Gleason score, and pathology TNM staging. The variants regarding the patients' incisional hernia included incisional hernia occurrence time after RARP, defect size, operation time, console time, blood loss, and follow-up time after the herniation occurrence. Furthermore, we established a defect size of $3 \times 2 \mathrm{~cm}^{2}$ as the cutoff value for using mesh reinforcement or not. Results: The mean defect area was $27.7 \mathrm{~cm}^{2}$, and the average operative time was $114.8 \mathrm{~min}$, with a mean console time of $87 \mathrm{~min}$.
\end{abstract}

This article is freely accessible online.

Correspondence to: Yen-Chuan Ou, MD, Ph.D., Deputy Superintendent, Professor in the Department of Urology of Tungs' Taichung Metro Harbor Hospital, No.699, Sec. 8, Taiwan Blvd. Taichung City 435, Taiwan, R.O.C. Tel: +886 0426581919 \#4153, Fax: +886 0426581155, Mob: +886 926918348, e-mail: ycou228@gmail.com

Key Words: Incisional hernia repair, laparoscopic, radical prostatectomy, robotics.
Blood loss was $32.5 \mathrm{ml}$, and the hospital stay for all patients was 3 days without complications. The mean follow-up period was 29.5 months, with no recurrence. Conclusion: RIHR is a feasible surgical method that is not inferior to the traditional open or laparoscopic repair. Furthermore, RIHR can possibly lessen the burden of both the surgeon and patient.

With the growing prevalence of prostate cancer (1), prostatectomy has become a vital surgery. At the same time, the use of robotic-assisted procedures has gained greater acceptance in various surgeries, and robotic-assisted radical prostatectomy (RARP) has become one of the most adopted procedures (2). The pentafecta of RARP for localized prostate cancer is excellent in experienced hands (3). However, some inevitable complications and severe sequela may occur $(4,5)$ : for example, trocar site infection and herniation after surgery, especially at the site of the umbilical wound where the RARP specimen was removed (4).

According to previous studies, the incisional hernia incidence rate is higher after RARP than with traditional open radical prostatectomy (6). Furthermore, current studies suggest that an incisional hernia could be repaired with either an open or laparoscopic method, both of which showed similar outcomes (7). Nevertheless, there are no clinical studies discussing robotic-assisted incisional hernia repair (RIHR), which turned out to show better results than the laparoscopic repair method in a simulated model (8).

As a result, it is reasonable to state that robotic-assisted incisional hernia repair (RIHR) has the potential to become widely used in the future. The goal of this study was to propose a 3-port surgical approach using da Vinci ${ }^{\circledR}$ robotics and previous port locations for surgical access to lessen the patient's recovery burden. This study aims to provide preliminary results of RIHR. 

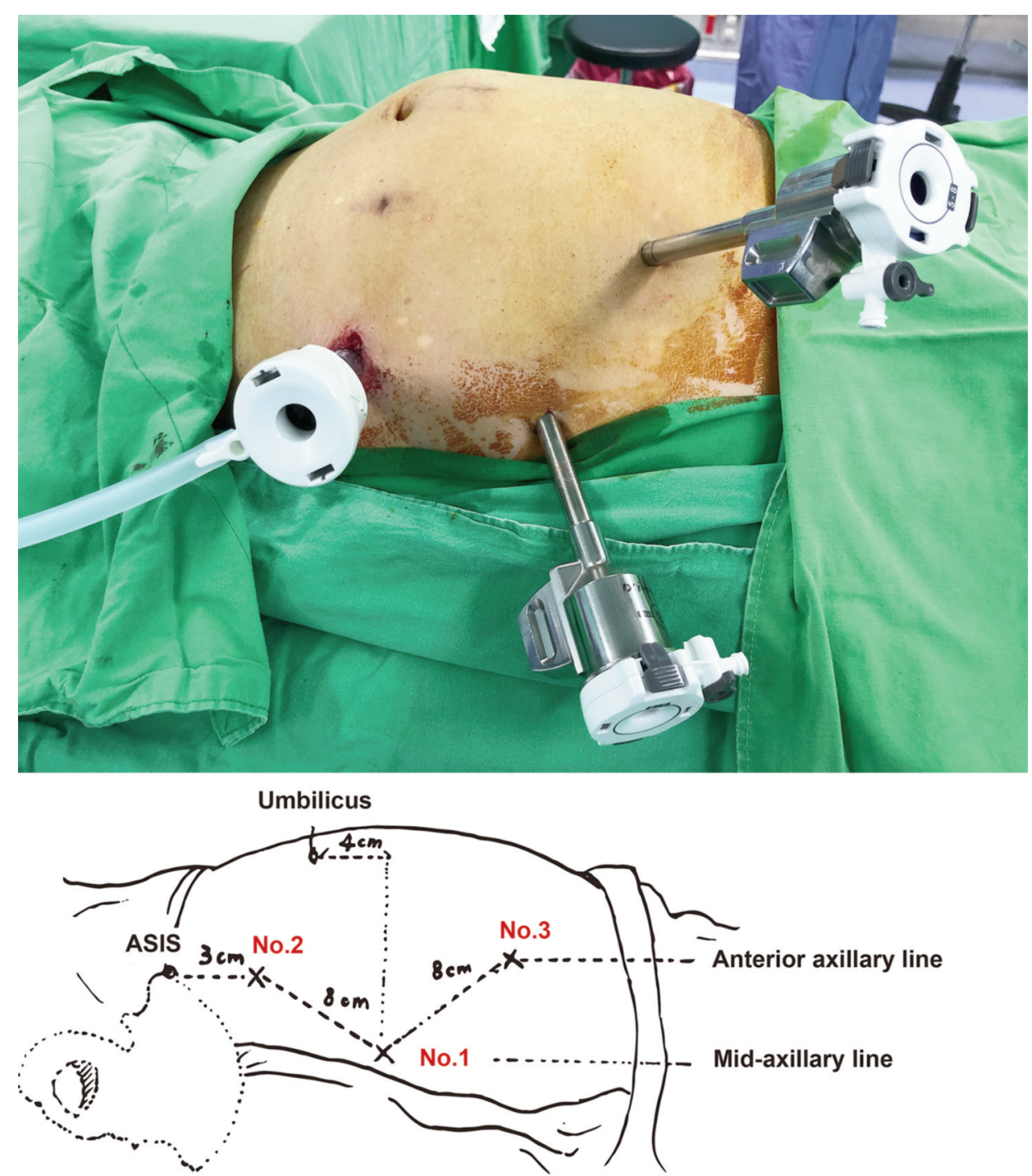

Figure 1. We sequentially located the camera site and the left and right instrument sites in a triangular position using the patient's umbilicus and ASIS as landmarks. After insertion of the camera port, a pneumo-peritoneum with pressure of 12-15 $\mathrm{mm} \mathrm{Hg}$ was first created by $\mathrm{CO}_{2}$ insufflation.

\section{Patients and Methods}

Patient recruitment. In this retrospective case series study, we focused on an observation group that consisted of 2,000 patients who underwent RARP performed by a single high-volume robotic surgeon (Y.C. OU) from December 2005 to June 2020, and the majority of patients maintained follow-up after surgery. We recruited 10 of the patients who experienced incisional herniation and underwent robotic hernia repair using the da Vinci Si or Xi robotic system (Surgical Intuitive, Sunnyvale, CA, USA) between February 2015 and June 2020.

The distinct patient variables evaluated in this study included age, body mass index (BMI), prostate specific antigen (PSA) level, pathology Gleason score, and pathology Tumor, Lymph node, Metastasis (TNM) staging. The parameters for the herniation repair included incisional hernia occurrence time after RARP, defect size, operation time, console time, blood loss, and follow-up time after the herniation occurrence.

Surgical technique. The patient was placed in the supine position, arms tucked in to the sides. Beanbag and shoulder pads can be used to protect the patient. Trocar porting was carried out at the patient's left lateral abdominal area. We first located a point that was at the intersection of 2 lines. The horizontal line was at the level of $4 \mathrm{~cm}$ superior to the umbilical, and the mid-axillary line was the vertical line. We used an 8 -mm trocar to set port No.1 (camera port), and $\mathrm{CO}_{2}$ insufflation with pressure set at $12-15 \mathrm{~mm}$ $\mathrm{Hg}$ was used to create a pneumo-peritoneum. Then, we used an 8$\mathrm{mm}$ trocar to set port No. 2 along the ASIS vertical line at a point that was $8 \mathrm{~cm}$ apart from port No.1. In fact, ports No.1 and No.2 were at the operative scar of the previous RARP. Last, we used an 8 -mm trocar to set port No.3 on the anterior axillary line at a point 

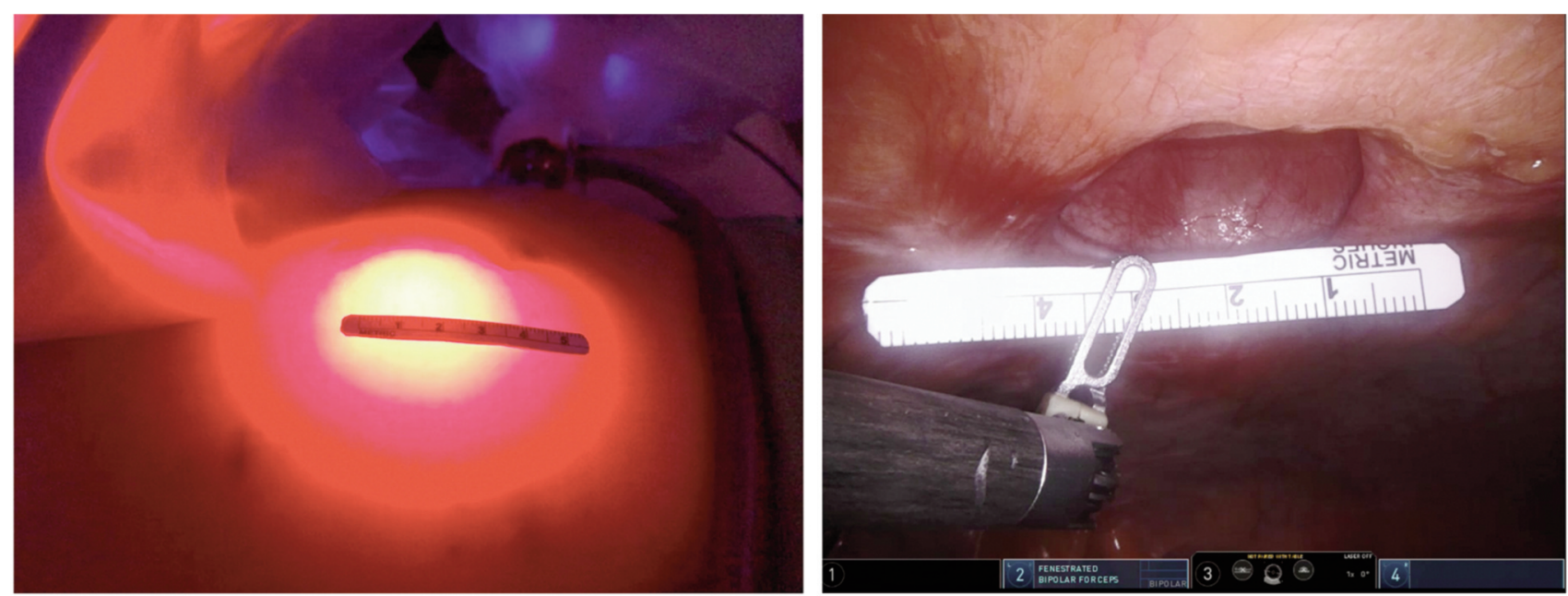

Figure 2. Measurement of the hernia defect size from the outside and inside of the patient's abdomen using a ruler.

that was also $8 \mathrm{~cm}$ apart from port No.1, to form a triangular position (Figure 1).

During surgery, port No.1 was the camera port. Port No.2 was the robotic left working port, and we mainly chose EndoWrist Fenestrated Bipolar Forceps for both the forceps and coagulation function. Port No.3 was a hybrid port which would accommodate both a right-hand instrument and the assistant's laparoscopic instruments. In this port, we used an Endowrist Large SutureCut Needle Driver for needle holding and cutting use, while the laparoscopic instrument could deliver mesh or suture, if needed. At the beginning, all of the patients' herniation defects were measured from both outside and inside the abdominal wall, with camera illumination (Figure 2). After identifying the fascial defect, we used the measurement of the defect to assist in further treatment decisions: if the defect was smaller than or equal to $6 \mathrm{~cm}^{2}$, for example $3 \times 2 \mathrm{~cm}^{2}$, as we proposed, direct closure could be enough. However, if the defect was larger than $3 \times 2 \mathrm{~cm}^{2}$, repairing with mesh (Bard ${ }^{\circledR}$ Composix $^{\text {TM }}$ L/P Mesh) was highly suggested. Moreover, we could use Covidien V-Loc ${ }^{\mathrm{TM}} 15 \mathrm{~cm}$ 2-0 sutures for re-approximation first to ensure the quality of the repair. For mesh localization in larger defects, we adopted a chandelier method that placed the Ethicon Vicryl violet CT-1 taper 2-0, approximately 12 $\mathrm{cm}$ in length, at the center of the mesh, and hooked the other end to the center of the defect (Figure 3). Afterwards, we lessened pneumo-peritoneum pressure to $8 \mathrm{~mm} \mathrm{Hg}$ and the edge of the defect was sutured to the rim of the mesh with Covidien V-Loc ${ }^{\mathrm{TM}} 15 \mathrm{~cm}$ 2-0 sutures (Medtronic, London, UK), using a GS-21 taper needle for continuous running sutures. An optional Weck ${ }^{\circledR}$ Hem-o-lok ${ }^{\circledR} 2$ 0 (Teleflex, NC, USA) was added for stability if needed. Otherwise, as the defect was of a small size $\left(6 \mathrm{~cm}^{2}\right)$, we simply used 2-0 V-Loc for circumferential suturing over the fascia to close the defect. In both situations, no drainage tube was placed and the trocar sites were closed carefully.

\section{Results}

A total of 10 patients were included in the study (Table I). The mean age was 70.3 years, and the average BMI was $26.9 \mathrm{~kg} / \mathrm{m}^{2}$.
Table I. Comparison of preoperative clinical characteristics of 10 cases who underwent robotic-assisted hernia repair.

\begin{tabular}{lccccc}
\hline Case No. & Age & $\begin{array}{c}\text { Body } \\
\text { mass index } \\
\text { (BMI) }\end{array}$ & $\begin{array}{c}\text { Prostate } \\
\text { specific } \\
\text { antigen (PSA) }\end{array}$ & $\begin{array}{c}\text { Pathology } \\
\text { Gleason } \\
\text { score }\end{array}$ & Staging \\
\hline 1 & 65 & 25.39 & 7.14 & $3+4=7$ & T3a \\
2 & 72 & 28.12 & 15.98 & $4+5=9$ & T3a \\
3 & 65 & 27.01 & 7.67 & $4+3=7$ & T2b \\
4 & 78 & 26.43 & 74.00 & $4+5=9$ & T3b \\
5 & 64 & 31.22 & 3.56 & $3+3=6$ & T1b \\
6 & 68 & 24.39 & 5.30 & $3+4=7$ & T2c \\
7 & 63 & 27.88 & 54.09 & $4+3=7$ & T3b \\
8 & 74 & 24.96 & 26.10 & $4+5=9$ & T2b \\
9 & 71 & 25.35 & 9.05 & $3+4=7$ & T1b \\
10 & 83 & 28.28 & 18.07 & $4+3=7$ & T3a \\
Mean & 70 & 26.90 & 22.10 & 7.5 & \\
Median & 70 & 26.72 & 12.52 & 7 & \\
SD & 7 & 2.05 & 23.61 & 1.08 & \\
Minimum & 63 & 24.39 & 3.56 & 6 & \\
Maximum & 83 & 31.22 & 74.00 & 9 & \\
\hline
\end{tabular}

All patients experienced incisional hernia after RARP, and all underwent hernia repair using the da Vinci ${ }^{\circledR}$ robotic system.

The average defect size of the herniation was $27.7 \mathrm{~cm}^{2}$. All 10 patients underwent robotic herniation repair. Seven of the 10 patients underwent repair with mesh, and 3 were sutured without mesh, as the defect size was smaller than or equal to $6 \mathrm{~cm}^{2}$. We chose Bard $^{\circledR}$ Composix ${ }^{\mathrm{TM}} \mathrm{L} / \mathrm{P}$ Mesh for these procedures. The average operative time was 114.8 min, with an average console time of $87 \mathrm{~min}$. Estimated blood loss during surgery was $32.5 \mathrm{ml}$. The blood loss for 7 patients was less than $5 \mathrm{ml}$, but we calculated it as $5 \mathrm{ml}$ to account for the actual undetected blood loss. We 

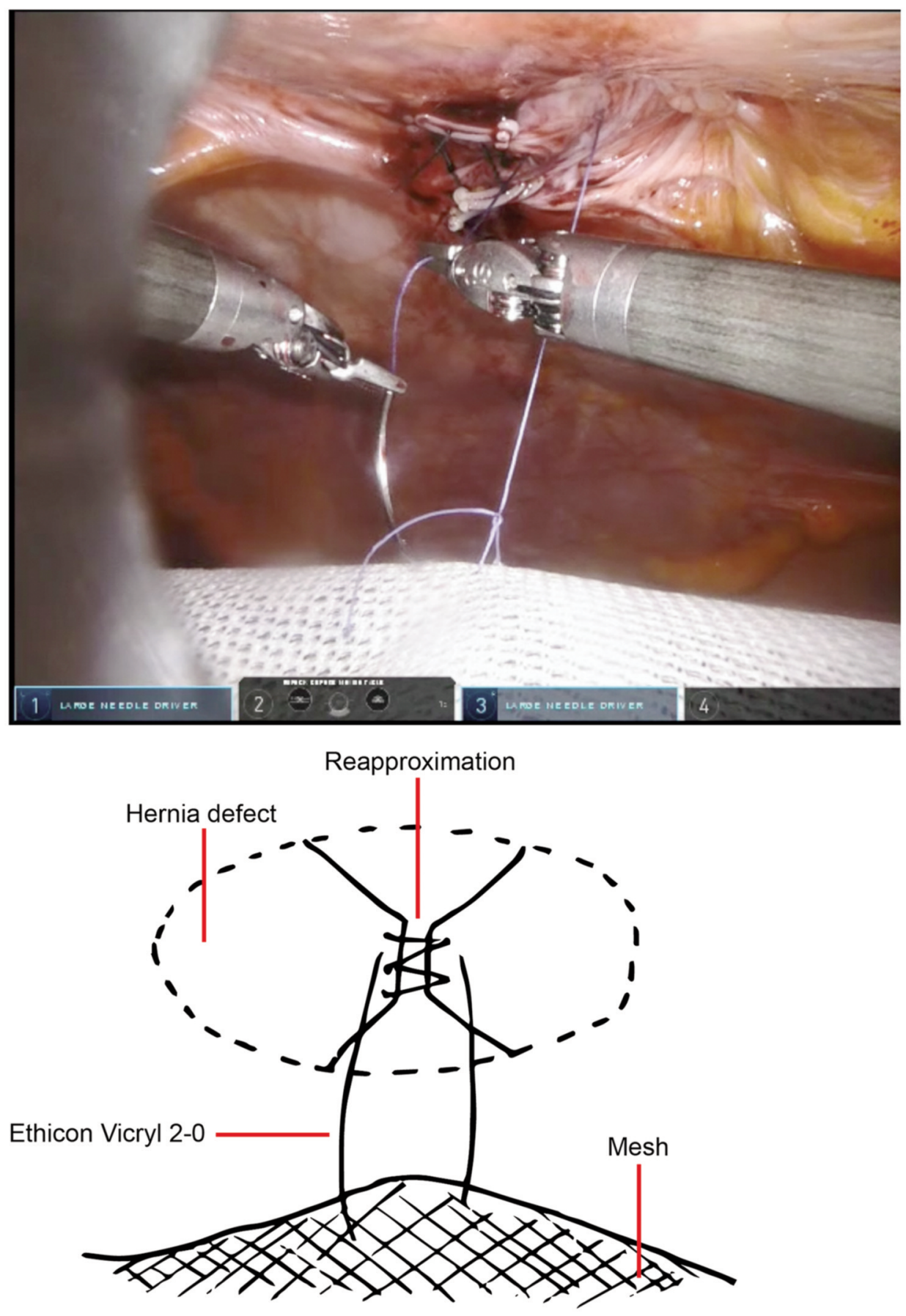

Figure 3. For a larger defect, we first re-approximated the defect. Then, we used the chandelier method to hook both the center of the defect and the mesh with Ethicon Vicryl 2-0.

encouraged ambulation as soon as possible after surgery, once the patient had clear consciousness. The mean hospital stay was 3 days with the following clinical pathway: the first day was for pre-operative preparation, the second day for the RIHR procedure, and the third day for discharge, if appropriate. All 10 patients were discharged without any complications, such as infection, hemorrhage or urine retention, on postoperative day 1 .

Before discharge, we educated the patients on activity restrictions, such as avoiding lifting things heavier than 4.5 $\mathrm{kg}$ for at least 6 weeks after the operation. An abdominal binder was routinely used for the patient's comfort (9). After 
Ou et al: Robotic Incisional Hernia Repair After Robotic-assisted Radical Prostatectomy

Table II. Characteristics of the 10 incisional hernia repair procedures.

\begin{tabular}{|c|c|c|c|c|c|c|c|}
\hline Case No. & $\begin{array}{c}\text { Defect area, } \\
\text { length } \times \text { width }\left(\mathrm{cm}^{2}\right)\end{array}$ & $\begin{array}{c}\text { Occurrence } \\
\text { timing (months) }\end{array}$ & $\begin{array}{l}\text { Operation time } \\
(\min )\end{array}$ & $\begin{array}{l}\text { Console time } \\
\text { (min) }\end{array}$ & $\begin{array}{l}\text { Blood loss } \\
\quad(\mathrm{ml})\end{array}$ & Mesh & $\begin{array}{l}\text { Follow-up time } \\
\text { (months) }\end{array}$ \\
\hline 1 & $5 \times 4=20$ & 12 & 70 & 45 & $<5$ & $15 \times 10$ & 48 \\
\hline 2 & $7 \times 5=35$ & 4 & 210 & 180 & 140 & $15 \times 10$ & 48 \\
\hline 3 & $3 \times 2=6$ & 4 & 218 & 180 & 100 & - & 38 \\
\hline 4 & $5 \times 4=20$ & 9 & 150 & 120 & 50 & $10 \times 15$ & 38 \\
\hline 5 & $5 \times 4=20$ & 6 & 95 & 65 & $<5$ & $10 \times 15$ & 48 \\
\hline 6 & $13 \times 8=104$ & 5 & 95 & 65 & $<5$ & $15.9 \times 21$ & 28 \\
\hline 7 & $6 \times 5=30$ & 5 & 95 & 75 & $<5$ & $15 \times 10$ & 30 \\
\hline 8 & $3 \times 2=6$ & 6 & 45 & 20 & $<5$ & - & 25 \\
\hline 9 & $5 \times 6=30$ & 6 & 80 & 50 & $<5$ & $15 \times 10$ & 7 \\
\hline 10 & $3 \times 2=6$ & 2 & 90 & 70 & $<5$ & - & 5 \\
\hline Mean & 27.7 & 6 & 115 & 87 & 32.5 & & 31.5 \\
\hline Median & 20 & 6 & 95 & 68 & 5 & & 34 \\
\hline SD & 28.3 & 3 & 59 & 55 & 49.1 & & 16 \\
\hline Minimum & 6 & 2 & 45 & 20 & 5 & & 5 \\
\hline Maximum & 104 & 12 & 218 & 180 & 140 & & 48 \\
\hline
\end{tabular}

discharge, all the patients maintained regular follow-up at the OPD every 2 months, with a mean follow-up period of 31.5 months. There has been no recurrence, as of this writing.

\section{Discussion}

This is the first clinical study to discuss the outcome of incisional repair using da Vinci ${ }^{\circledR}$ robotics. Our case series showed promising results with no recurrence and a relatively lower complication rate than previous studies of traditional and laparoscopic methods (10). Furthermore, blood loss and hospital stay were not significantly different from that of the 2 other methods. However, operation time was longer if the surgical port set-up time was included. Nevertheless, by dividing the patient group into first and second halves, we could see that there was a significant learning curve. Operation time for the first 5 patients was $148.6 \mathrm{~min}$, and that for the second 5 patients was $81 \mathrm{~min}$.

A previous study using a training model implied that RIHR could be better than the laparoscopic method in terms of reducing the mental and physical effort of the surgeons (8). We propose using RIHR as a routine method, since no significant disadvantage was found and its use could possibly provide a better working experience for the surgeon.

For the standard for mesh reinforcement, we proposed $3 \times 2$ $\mathrm{cm}^{2}$ as a cutoff value for several reasons. First, as the previous literature suggests, incisional hernia repair in all patients ought to be done with mesh (11). However, other studies state that mesh usage is related to a higher rate of infection or late-onset adhesion $(12,13)$. As a result, we do not routinely use mesh with all defect sizes so as to lessen the overall complication rate. Second, we propose that direct closure of a smaller defect is safer when the defect is more oval, rather than a circular shape. That is, the width of the defect should not be longer than $2 \mathrm{~cm}$, based on the surgeon's experience with previous herniation repair cases and the situation each time.

And for suturing, we used the slowly-absorbable (180 days) Covidien V-Loc ${ }^{\mathrm{TM}} 15 \mathrm{~cm} \mathrm{2-0}$ sutures for fascia closure based on a previous meta-analysis of midline abdominal incisions that suggests slowly-absorbable sutures to be more preferable than non-absorbable ones $(12,14)$.

Moreover, the 3-port approach can be achieved using multi-functional instruments and hybrid ports with laparoscopic instruments delivering required materials such as sutures or mesh into the abdomen. With this, we reduced the number of trocar sites, wound healing time, and efforts by the patients. In our experience, this approach has not caused any additional iatrogenic surgical complications due to the fewer ports and the subsequently limited visual field.

Last but not least, previous studies revealed a higher rate of incisional hernia after RARP than after the open method $(4,13)$. Among the 2,000 patients who underwent RARP from a single doctor (Y.C. OU), the incidence of incisional hernia was only $0.9 \%(18 / 2000)$. While the 18 patients included 10 patients who underwent robotic-assisted incisional hernia repair and 8 patients opted for traditional open-method repair. Hence, we suggest that the incidence rate could be lower with RARP if it is performed by a highvolume surgeon. Furthermore, a study suggested that horizontal closure of the fascia could significantly reduce the complication rate of incisional hernia (15). However, in our study, we still followed the old way by vertically suturing the fascia, and it turned out that there was no difference in the post-RARP incisional hernia rate in when the procedure was performed by an experienced surgeon. 
In this study, we reported a 3-port approach that can lessen the operational burden for the patients. All operations were performed by a single doctor, which could therefore reduce the technique bias due to individual differences. And, we presented another option for incisional hernia repair, with the hope that this could promote more studies in robotic-assisted hernia repair. Nevertheless, we acknowledge the limitations of this study, including a patient number too small to establish statistically valid evidence for the outcomes of this surgical approach. Another factor that cannot be neglected is the higher cost for da Vinci ${ }^{\circledR}$ system. However, we regard RIHR to be an effective and safe procedure that has a potential to develop its own advantages over traditional approaches.

\section{Conclusion}

In patients who have experienced post-RARP incisional hernia, the adoption of robotic-assisted hernia repair surgical approach showed fulfilling results in various aspects.

\section{Conflicts of Interest}

The Authors have no affiliation with any organization with a direct or indirect financial interest in the subject matter discussed in the manuscript. There are no conflicts of interest.

\section{Authors' Contributions}

Design and conception: YCO, HCO. Drafting of manuscript: HCO, YCO. Acquisition of data: LHH, MCT, WCW, TYT. Critical revision: CYH, YSL, CHL. Statistical analysis: KHC.

\section{References}

1 Taitt HE: Global trends and prostate cancer: A review of incidence, detection, and mortality as influenced by race, ethnicity, and geographic location. Am J Mens Health 12(6): 1807-1823, 2018. PMID: 30203706. DOI: 10.1177/ 1557988318798279

2 Du Y, Long Q, Guan B, Mu L, Tian J, Jiang Y, Bai X and Wu D: Robot-assisted radical prostatectomy is more beneficial for prostate cancer patients: A system review and meta-analysis. Med Sci Monit 24: 272-287, 2018. PMID: 29332100. DOI: 10.12659/msm.907092

3 Ou YC, Yang CK, Kang HM, Chang KS, Wang J, Hung SW, Tung MC, Tewari AK and Patel VR: Pentafecta outcomes of 230 cases of robotic-assisted radical prostatectomy with bilateral neurovascular bundle preservation. Anticancer Res 35(9): 50075013, 2015. PMID: 26254400.

4 Ou YC, Yang CK, Chang KS, Wang J, Hung SW, Tung MC, Tewari AK and Patel VR: Prevention and management of complications during robotic-assisted laparoscopic radical prostatectomy following comprehensive planning: A large series involving a single surgeon. Anticancer Res 36(4): 1991-1998, 2016. PMID: 27069192.
5 Hung CF, Yang CK, Cheng CL and Ou YC: Bowel complication during robotic-assisted laparoscopic radical prostatectomy. Anticancer Res 31(10): 3497-3501, 2011. PMID: 21965768.

6 Hermann M, Gustafsson O and Sandblom G: Incidence of incisional hernia after minimally invasive and open radical prostatectomy: A population-based nationwide study. Scand J Urol 51(4): 264-268, 2017. PMID: 28398125. DOI: $10.1080 / 21681805.2017 .1301991$

7 Al Chalabi H, Larkin J, Mehigan B and McCormick P: A systematic review of laparoscopic versus open abdominal incisional hernia repair, with meta-analysis of randomized controlled trials. Int J Surg 20: 65-74, 2015. PMID: 26074289. DOI: $10.1016 /$ j.ijsu.2015.05.050

8 Sánchez A, Rodríguez O, Jara G, Sánchez R, Vegas L, Rosciano $\mathrm{J}$ and Estrada L: Robot-assisted surgery and incisional hernia: A comparative study of ergonomics in a training model. J Robot Surg 12(3): 523-527, 2018. PMID: 29302848. DOI: 10.1007/ s11701-017-0777-y

9 Trujillo CN, Fowler A, Al-Temimi MH, Ali A, Johna S and Tessier D: Complex ventral hernias: A review of past to present. Perm J 22: 17-015, 2018. PMID: 29272245. DOI: 10.7812/ tpp/17-015

10 Eker HH, Hansson BM, Buunen M, Janssen IM, Pierik RE, Hop WC, Bonjer HJ, Jeekel J and Lange JF: Laparoscopic vs. open incisional hernia repair: A randomized clinical trial. JAMA Surg 148(3): 259-263, 2013. PMID: 23552714. DOI: 10.1001/ jamasurg.2013.1466

11 Breuing K, Butler CE, Ferzoco S, Franz M, Hultman CS, Kilbridge JF, Rosen M, Silverman RP and Vargo D: Incisional ventral hernias: Review of the literature and recommendations regarding the grading and technique of repair. Surgery $148(3)$ : 544-558, 2010. PMID: 20304452. DOI: 10.1016/j.surg. 2010.01 .008

12 El-Gazzaz G, Erem HH, Aytac E, Salcedo L, Stocchi L and Kiran RP: Risk of infection and hernia recurrence for patients undergoing ventral hernia repair with non-absorbable or biological mesh during open bowel procedures. Tech Coloproctol 17(3): 315-320, 2013. PMID: 23179894. DOI: 10.1007/s10151-012-0928-0

13 Bensley RP, Schermerhorn ML, Hurks R, Sachs T, Boyd CA, O'Malley AJ, Cotterill P and Landon BE: Risk of late-onset adhesions and incisional hernia repairs after surgery. J Am Coll Surg 216(6): 1159-1167, 1167 e1151-1112, 2013. PMID: 23623220. DOI: 10.1016/j.jamcollsurg.2013.01.060

14 van 't Riet M, Steyerberg EW, Nellensteyn J, Bonjer HJ and Jeekel J: Meta-analysis of techniques for closure of midline abdominal incisions. Br J Surg 89(11): 1350-1356, 2002. PMID: 12390373. DOI: 10.1046/j.1365-2168.2002.02258.x

15 Liss MA, Skarecky D, Morales B, Osann K, Eichel L and Ahlering TE: Preventing perioperative complications of roboticassisted radical prostatectomy. Urology 81(2): 319-323, 2013. PMID: 23374792. DOI: 10.1016/j.urology.2012.09.033

Received June 30, 2020

Revised July 21, 2020

Accepted July 22, 2020 